\title{
EQUICONTINUITY AND INDIVISIBILITY IN TRANSFORMATION GROUPS
}

\author{
BY
}

\section{PING-FUN LAM}

\begin{abstract}
A notion called indivisibility for transformation groups is studied in some detail. The proofs of some theorems announced in [10] are given. Certain types of nonequicontinuous sets which do not disconnect the ir metric continuum phase spaces and which were not known to exist previously are exhibited.
\end{abstract}

0 . Introduction. Let $X$ be either a metric space or a uniform $T_{2}$ space. Consider a topological transformation group $(X, T, \pi)$ in the sense of Gottschalk and Hedlund [4]. If we assume that the group of transformations is either uniformly equicontinuous or pointwise equicontinuous, it is well known that various interesting results can be obtained. For instance, if $X$ is compact then the equicontinuity property is equivalent to uniform almost periodicity $[4,4.38]$ and as a result the space is decomposed into the union of minimal sets each of which carries the structure of a coset space of a compact group. Transformation groups without the equicontinuity property are often studied by considering its representations on those which have this property. The approach that we introduce in this paper is somewhat different. We use a notion called indivisibility (cf. (2.2)) which displays some weak asymptoticity relations among subsets of $X$. For a fixed transformation group the class of indivisibilities involves three variables and a condition. Similar to the notion of connectedness, each indivisibility decomposes a subspace of $X$ into equivalence classes which we call indivisible components (cf. (2.5)). We may think of it in the following way: if two points are sitting in two different indivisible components then the actions on them have dissimilarity of certain kind. We will follow this global scheme but limit ourselves to certain indivisibilities which can give the topological nature of the set of nonequicontinuous points $N(X)$. A typical application is given in Theorem 2 of [10] which states that if $X$ is a connected compact metric space and $N(X)$ is 0 -dimensional and if a certain condition on indivisibility is satisfied, then $N(X)$ is either a Cantor set or it consists of at most two points. Some details of applications of this work are given in [11]. The approach through the notion of indivisibility enables [11] to extend and to

Received by the editors August 16, 1971.

AMS (MOS) subject classifications (1969). Primary 5482.

Key words and phrases. Equicontinuity, upper semicontinuous function, co-meager set, minimal set, one-point compactification, scattered set, semicontinuum, zero-dimensional set, Peano curve. 
clarify the earlier work on nearly equicontinuous transformation groups by Homma and Kinoshita [6], Kaul [7] and Gray and Roberson [5].

Contents. In $\oint_{1}$ we show that $N(X)$ is always a union of closed invariant sets. In $\S 2$ an axiomatic study of indivisibility is given. In $\S 3$ some selected classes of indivisibilities and their special properties are considered. Topological sufficient conditions for indivisibilities of $\$ 3$ are given in $\S 4$. The sharpness of such sufficient conditions is justified in $\$ 5$ by counterexamples. The counterexamples also exhibit some new transformation groups in terms of the set $N(X)$. For instance Example 5.4 gives a continuous flow on a metric continuum such that the set $N(X)$ is countably infinite and does not disconnect the space. The previously known $N(X)$ which does not disconnect a continuum is either finite or uncountable (cf. [5], [6], [7], [10]).

Some theorems announced in [10] are given proofs in this paper. Theorem 1 is proved in (1.4), Theorem 3 is proved in $\$ 4$ and part of Theorem 4 is proved in (5.3). The rest of the theorems announced in [10] will be proved in [11] and another forthcoming paper.

By abuse of language we will use the word indivisibility both in the abstract sense as a notion and in the concrete sense as an object. To be general, we present this work in terms of uniform $T_{2}$ phase spaces.

Standing hypothesis. All transformation groups $(X, T, \pi)$ will be assumed to have uniform $T_{2}$ space $X$. Unless explicitly stated otherwise, the transformation group $\xi=(X, T, \pi)$ and its uniformity will generally be fixed.

Standing notation.

(1) 2 = the class of all nets in $T$.

(2) $\mathscr{Q}_{0}=$ the set of all sequences in $T$.

(3) $\mathcal{P}(Z)=$ the set of all subsets of $Z, Z$ a set.

(4) $U=$ the uniformity of $X$.

(5) $S^{*}=S \cup\{\infty\}$, the one-point compactification of a topological space $S$.

(6) $x \alpha$ : if $x \in X$ and $\alpha=\left\{t_{i}\right\} \in \mathcal{Q}$ then $x \alpha$ denotes the net $\left\{x t_{i}\right\}$ in $X$.

(7) The word "family" will be used for both a set and a class. The involvement of logic, however, is minimal.

(8) $E(X)=$ the set of all equicontinuous points of $(X, T, \pi), N(X)=X-E(X)$ $=$ the set of all nonequicontinuous points of $(X, T, \pi)$ (cf. (1.1)).

(9) A set of standing notation involving indivisibilities will be given in (3.1).

The main reference for transformation groups is Gottschalk and Hedlund [4] and the main reference for topology is Kuratowski [9].

1. Nonequicontinuous set is a union of closed invariant sets. We recall a well-known definition.

1.1. Definition. A point $x \in X$ is said to be equicontinuous if for every $u \in \mathcal{U}$ there exists $v \in \mathcal{U}$ such that 


$$
(x, y) \in v, y \in X \Rightarrow(x t, y t) \in u, \text { for all } t \in T .
$$

Otherwise, $x$ is said to be nonequicontinuous.

When $X$ is a metric space, we take $\mathcal{U}$ to be the uniformity generated by the metric $d$ of $X$. Then clearly $x \in X$ is equicontinuous iff for every $\epsilon>0$ there exists $\delta>0$ such that

$$
d(x, y)<\delta, y \in X \Rightarrow d(x t, y t)<\epsilon, \text { for all } t \in T \text {. }
$$

$E(X)$ or $E(X, \mathcal{U}, T)$ will denote the set of all equicontinuous points, $N(X)$ or $N(X, \mathcal{U}, T)$ will denote the set of all nonequicontinuous points. It is easily verified that $E(X)$ and $N(X)$ are invariant under $T$. In general one of the two sets may be void. In fact if $(X, T, \pi)$ is uniformly almost periodic and $X$ is compact, then $N(X)=\varnothing ;$ if $(X, T, \pi)$ is expansive and $X$ is self-dense, then $E(X)=\varnothing$. Moreover, the sets $E(X)$ and $N(X)$ depend on $\mathcal{U}$ in case $X$ is not compact.

Every nonempty closed invariant set $F$ confines the position of orbit closures $\mathrm{Cl}(x T)$, in particular minimal sets, in the sense that if $x \in F$ then $\mathrm{Cl}(x T) \subset F$. We will need this property for $N(X)$. In general $N(X)$ is not a closed set. However, the property above holds for nonempty sets $F$ which can be expressed as a union of closed invariant sets.

1.2. Theorem. The set $N(X)$ is a union of closed invariant sets. In fact the closed invariants sets are $F_{u}(X)=\{x \in X \mid$ every neighborbood $V$ of $x$ satisfies $V t \times V t \not \subset u$ for some $\left.t=t_{V} \in T\right\}, u \in \mathcal{U}$.

Proof. The proof is very simple. A rigorous argument can be given as follows. Consider the product transformation group $(\hat{X}, T, \hat{\pi})$, i.e. $\hat{X}=X \times X$ with product topology and $\hat{\pi}=\hat{X} \times T \rightarrow \hat{X}$ is defined by

$$
((x, y), t) \hat{\pi}=\left((x, t)_{\pi},(y, t)_{\pi}\right), \quad x, y \in X, t \in T .
$$

There exists a natrual transformation group isomorphism of $(X, T, \pi)$ onto $(\Delta, T, \hat{\pi})$ where $\Delta$ is the diagonal of $\hat{X}$. The image of $F_{u}(X)$ under this isomorphism is the set $\Delta \cap \mathrm{Cl}[(\hat{X}-u) T]$. It follows easily that $F_{u}(X)$, is closed, invariant and $N(X)=\bigcup\left\{F_{u}(X) \mid u \in \mathcal{U}\right\}$.

If $X$ is a metric space, then $\mathcal{U}$ has a countable base, hence $N(X)$ is a countable union of closed invariant sets. We will give a different proof of this fact by making use of an upper semicontinuous function $f_{T}: X \rightarrow[0, \infty]$, which we called the spread function of $(X, T, \pi)[10]$. By definition

$$
x f_{T}=\inf \left\{\sup \left\{\text { diameter }\left(S_{\epsilon}(x) t\right) \mid t \in T\right\}, \epsilon>0\right\}, \quad x \in X,
$$

where $S_{\epsilon}(x)$ is the $\epsilon$-open ball around $x$. The function simplifies the expression of $F_{u}(X)$. In the opinion of the author, the function $f_{T}$ perhaps has important significance beyond this study. 
1.3. Lemma. Let $f_{T}$ be the spread function for metric phase space $X$. Then

(1) $f_{T}$ is upper semicontinuous.

(2) $(x t) f_{T}=x f_{T}$; for all $x \in X, t \in T$.

(3) If $x \in X$, then $x \in N(X)$ iff $x f_{T} \neq 0$.

1.4. Theorem. If $X$ is a metric space, then $N(X)$ is a countable union of closed invariant sets.

Proof. The proof follows directly from the three properties of the lemma. From (1) we have that $([c, \infty]) f_{T}^{-1}$ is a closed set for every $c, 0 \leq c \leq \infty$. From (2) the sets are invariant and from (3) we have

$$
N(X)=((0, \infty]) f_{T}^{-1}=\bigcup_{n=1}^{\infty}([1 / n, \infty]) f_{T}^{-1} \quad(n \text { : integers }) .
$$

Theorem 1.4 is the Theorem 1 given in [10].

1.5. Corollary. If $X$ is a metric space and $E(X)$ is dense, then $E(X)$ is a co-meager set (i.e. its complement is a set of first category).

We observe that the corollary can be obtained independently from the theorem. Under the hypothesis the set of discontinuities of $f_{T}$ is precisely $N(X)$. Now the set of discontinuities of an upper semicontinuous function is a set of first category [9, Vol. 1, p. 394, Theorem 1].

Corollary 1.5 has an important significance. It says that for the complete metric $X$ case, once $E(X)$ is dense, then $N(X)$ can be regarded as small. It rules out the possibility of constructing a transformation group on the plane so that $E(X)$ consists of all rational pairs. It also opens up the question of which subsets of $X$ are admissible as $E(X)$ for some acting group $T$.

1.6. Remark. Let $X$ be again a uniform $T_{2}$ space. Then

(1) $p \in N(X)$, then $\mathrm{Cl}(p T) \subset N(X)$.

(2) If $M$ is a minimal set, then either $M \subset N(X)$ or $M \subset E(X)$.

(3) If $X$ is a metric space, then $M f_{T}$ is a singleton for every minimal set $M$.

Proof. Statements (1) and (2) are immediate consequences of Theorem 1.2. Statement (3) can be easily obtained by contradiction.

Remark 1.6(2) says that if a point of a minimal set is equicontinuous, so must be all other points. This fact can be proved directly. The result for the case when $M \subset N(X)$ can be strengthened. Note that $N(X)$ is expressed as 'layers' $F_{u}(X)$. Hence if $u, v \in \mathcal{U}, M \cap F_{u}(X)=\varnothing, M \cap F_{v}(X) \neq \varnothing$, then $M \subset F_{v}(X)-F_{u}(X)$.

2. Indivisibility; general properties.

2.1. Definition. A $C$-object is defined as a 4 -tuple $(\xi, Y, \mathcal{F}, \mathcal{K})$ which has the following properties:

(1) $\xi=(X, T, \pi)$ is a transformation group, where $X$ is given a fixed uniformity. 
(2) $Y \subset X$.

(3) $\mathcal{F} \subset \mathcal{Q}$ (see standing notation).

(4) $K \subset \mathscr{P}(X)$.

2.2. Definition. A $C$-object $(\xi, Y, \mathcal{F}, \mathcal{K})$ is called an indivisibility if for every $a \in \mathcal{F}$ and for every $K \in \mathcal{K}$ whenever there exists $y_{0} \in Y$ such that the net $y_{0}$ a has a limit point in $K$, then the net $y \alpha$ has a limit point in $K$, for every $y$ $\epsilon Y$. A $C$-object which is not an indivisibility is cailed divisible.

Clearly a $C$-object $(\xi, Y, \mathcal{F}, \mathcal{K})$ is an indivisibility iff $(\xi, Y, a, K)$ are indivisibilities for all $\alpha \in \mathcal{F}$ and for all $K \in \mathcal{K}$ (a family which has exactly one element $m$ is denoted by $m$ as well as $\{m\}$ ). Other obvious properties of indivisibility are given as follows.

2.3. Proposition. Let $\sigma=(\xi, Y, \mathcal{F}, \mathcal{K})$ be a $C$-object. Then the following properties bold:

(1) If $Y$ is a point, then $\sigma$ is an indivisibility.

(2) If $\sigma$ is an indivisibility and $Y_{1} \subset Y$, then $\left(\xi, Y_{1}, \mathcal{F}, \mathcal{K}\right)$ is also an indivisibility.

(3) If $\sigma$ is an indivisibility and $\sigma^{\prime}=\left(\xi, Y^{\prime}, \mathcal{F}, \mathcal{K}\right)$ is an indivisibility, where $Y \cap Y^{\prime} \neq \varnothing$, then $\left(\xi, Y \cup Y^{\prime}, \mathcal{F}, \mathcal{K}\right)$ is an indivisibility.

(4) If $\left\{Y_{j}\right\}$ is an arbitrary family of subsets of $X$ totally ordered by inclusions and if all $\left(\xi, Y_{j}, \mathcal{F}, \mathcal{K}\right)$ are indivisibilities, then $\left(\xi, \cup_{j} Y_{j}, \mathcal{F}, \mathcal{K}\right)$ is an indivisibility.

(5) If $\sigma$ is an indivisibility and $\mathcal{F}_{1} \subset \mathcal{F}$, then $(\xi, Y, \mathcal{F}, \mathcal{K})$ is an indivisibility.

(6) If $\sigma$ is an indivisibility and $\mathcal{K}_{1} \subset \mathcal{K}$, then $\left(\xi, Y, \mathcal{F}^{1} \mathcal{K}_{1}\right)$ is an indivisibility.

(7) Let $\mathcal{K}=\left\{K_{j}\right\}$ and $L=\bigcup_{j} K_{j}$. If $\sigma$ is an indivisibility, then $(\xi, Y, \mathcal{F}, L)$ is an indivisibility.

In general one would expect that a given $C$-object may fail to be an indivisibility. In such a situation the $C$-object is resolved into, so called, indivisible components.

2.4. Proposition. Let $\sigma=(\xi, Y, \mathcal{F}, \mathcal{K})$ be a $C$-object. Then the following properties bold:

(1) Every $y \in Y$ lies in a subset $I_{y}=I_{y}(\sigma) \subset Y$ which is maximal with respect to the property that $\left(\xi, I_{y}, \mathcal{F}, \mathcal{K}\right)$ is an indivisibility.

(2) If $x \in I_{y}$, then $I_{x}=I_{y}$. If $x, y \in Y$ and $I_{x} \cap I_{y} \neq \varnothing$, then $I_{x}=I_{y}$. Hence the family $\left\{I_{y} \mid y \in Y\right\}$ forms a decomposition of the space $Y$.

(3) $I_{y}(\sigma)=\bigcap\left\{I_{y}(\xi, Y, \alpha, \mathcal{K}) \mid \alpha \in \mathcal{F}\right\}$.

Proof. For statements (1) and (2), apply (2.3(1), (3), (4)) and Zorn's lemma. Statement (3) follows from the remark before (2.3). 
2.5. Definition. A member of the decomposition of the $C$ object $\sigma$ in (2.4) is called an indivisible component of $\sigma$ or simply of $Y$.

We will see in the next section that, within the class of $C$-objects which we are interested in, the indivisible components simulate the usual topological com. ponents for being relatively closed in $Y$. In $\$ 4$ we will compare such indivisible components with the topological components.

2.6. Definition. An irreducible subnet of a divisible $C$-object $(\xi, Y, \mathcal{F}, \mathcal{K})$ is a net $\beta$ which has the following properties:

(1) $\beta$ is a subnet of some $\alpha \in \mathcal{F}$.

(2) $\lim x \beta$ exists in $X^{*}$, for every $x \in X$ (see standing notation).

(3) $(\xi, Y, \gamma, \mathcal{K})$ is divisible for every subnet $\gamma$ of $\beta$.

We will find the following reduction property very useful in the sequel.

2.7. Proposition, Every divisible C-object has an irreducible subnet.

Proof. The proposition is a simple consequence of the Tychonoff theorem on the product of compact spaces. If the $C$-object $(\xi, Y, \mathcal{F}, \mathcal{K})$ is divisible, then there exist $a \in \mathcal{F}$ and $K \in \mathcal{K}$ such that $(\xi, Y, a, K)$ is divisible. Then there exist points $y_{0}, y_{1} \in Y$ such that $y_{0}$ a has a limit point in $K$ and $y_{1}$ a has no limit point in $K$. We may assume that $\lim y_{0} \alpha=p \in K$ exists. Regarding each term of $\alpha$ as a map from $X$ to $X^{*}$ and applying the Tychonoff theorem we obtain a convergent subnet $\beta$. Then $\beta$ satisfies (1), (2) and (3).

Since $X^{*}$ is not yet a $T_{2}$-space, the $\operatorname{limit} \lim x \beta$ in (2.6(2)) may have more than one point. Later on we will assume that $X$ is locally compact and this defect will be overcome.

We will call a family $\mathcal{F} \subset \mathcal{Q}$ bereditary if whenever $\alpha \in \mathcal{F}$ then $\beta \in \mathcal{F}$ for every subnet $\beta$ of $\alpha$.

2.8. Corollary. Let $(\xi, Y, \mathcal{F}, \mathcal{K})$ be a $C$-object, where $\mathcal{F}$ is bereditary. Let $\mathfrak{F}_{c}$ denote the elements of $\mathcal{F}$ which converge pointwise in $X$ (to points in $X^{*}$ ). Then $(\xi, Y, \mathcal{F}, \mathcal{K})$ is an indivisibility $\Leftrightarrow\left(\xi, Y, \mathcal{F}_{c}, \mathcal{K}\right)$ is an indivisibility.

The family 2, for instance, is hereditary, while the subfamily $\mathscr{Z}_{0}$ is not. However, as one would expect, under suitable restrictions the effects of 2 and $2_{0}$ with respect to indivisibility are the same. We illustrate this fact by the following result.

2.9. Proposition. Let $X$ be a locally compact separable metric space, $K \subset$ $\mathscr{P}(X), Y \subset X$. Then $\sigma=(\xi, Y, \mathcal{2}, \mathcal{K})$ is an indivisibility $\Leftrightarrow\left(\xi, Y, \mathscr{Q}_{0}, \mathcal{K}\right)$ is an in. divisibility.

Proof. The $(\Rightarrow$ part follows from $(2.3(5))$.

$\Leftarrow$. By contradiction. Let $\beta$ be an irreducible subnet of $\sigma$ as given in (2.6). 
Then there exist $y_{0}, y_{1} \in Y$ such that $\lim y_{0} \beta=p \in K \in \mathcal{K}$ and $\lim y_{1} \beta=q \in X^{*}$ - K. Note now $X^{*}=X \cup\{\infty\}$ is a metric space. Out of $\beta$ one can easily find a sequence $\gamma \in \mathscr{Q}_{0}$ such that $\lim y_{0} \gamma=p$ and $\lim y_{1} \gamma=q$. We then have a contradiction.

Hereditary families are easy to deal with because of (2.8). As we see from (2.9) the results for such families can often be applied to nonhereditary families.

Another useful device for the later development is the extension of $(X, T, \pi)$ to its one-point compactification. We will do this regardless of whether $X$ is compact or not.

2.10. Proposition. If $X$ is locally compact, then there is a unique extension $\pi^{*}$ of the map $\pi$ of $\xi=(X, T, \pi)$ so that $\xi^{*}=\left(X^{*}, T, \pi^{*}\right)$ becomes a transforma. tion group.

Proof. Define $(\infty, t) \pi^{*}=\infty$ for all $t \in T$. The verification of the proposition is straightforward and is omitted.

Note that the space $X^{*}$, being compact, has a unique uniformity which is compatible with the topology. The set $E\left(X^{*}\right)$ and $N\left(X^{*}\right)$ for $\xi^{*}$ are then uniquely defined. The most important property of the extension from $\xi$ to $\xi^{*}$ is the following one.

2.11. Proposition. Let $X$ be locally compact. Then $E(X) \subset E\left(X^{*}\right)$.

Proof. It suffices to show that $N\left(X^{*}\right) \cap X \subset N(X)$. Thus let $\mathrm{U}^{*}$ be the uniformity of $X^{*}$. If $p \in N\left(X^{*}\right) \cap X$, then there exist $u^{*} \in \mathcal{U}^{*}$, nets $\left\{x_{i}\right\},\left\{y_{i}\right\}$ in $X$ and a net $\left\{t_{i}\right\}$ in $T$ which satisfy the following property:

$$
\lim y_{i}=p=\lim x_{i}, \quad\left(x_{i} t_{i}, y_{i} t_{i}\right) \notin u^{*} .
$$

By compactness of $X^{*}$ we may assume that $\left\{x_{i} t_{i}\right\}$ converges to $a \in X^{*}$ and $\left\{y_{i} t_{i}\right\}$ converges to $b \in X^{*}$. Then one of the points $a$ and $b$ is not $\infty$. We may assume that $b \neq \infty$. There exists $v \in \mathcal{U}$ such that $b v^{3} \subset b u^{*}$. Then $\left(b, x_{i} t_{i}\right) \notin v^{2}$ for sufficiently large $i$. For large $i$ we have that $\left(y_{i} t_{i}, x_{i} t_{i}\right) \notin v$, otherwise

$$
\left(b, x_{i} t_{i}\right)=\left(b, y_{i} t_{i}\right)\left(y_{i} t_{i}, x_{i} t_{i}\right) \in v^{2} .
$$

It follows that $p \in N(X)$.

3. Indivisibilities of equicontinuity type. In the remainder of the paper we will restrict our attention to a class of indivisibilities in which the notion of equicontinuity plays a crucial role. They are given as follows.

3.1. Definition. An indivisibility $\sigma=(\xi, Y, \mathcal{F}, \mathcal{K})$ is said to be of equicon. tinuity type of $\{$ first kind $\}$ second kind $\}$ third kind $\}$ if, respectively, $\{\mathcal{K}$ is the set of all components of $N(X)\}\{\mathcal{K}=\{E(X)\}\}\{\mathcal{K}=\{X\}\}$. We also say, respectively,

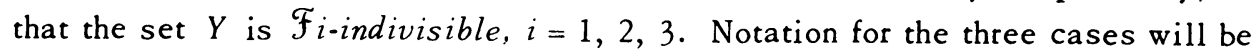


$\mathcal{F} \backslash Y, \mathcal{F} \not Y, \mathcal{F} \| Y$. The contracts will be denoted by $\mathcal{F} \mid Y, \mathcal{F}\|Y, \mathcal{F}\| Y$, respectively. The set $Y$ is said to be completely indivisible by $\mathcal{F}$ (notation $\mathfrak{F} \pitchfork Y$ ),

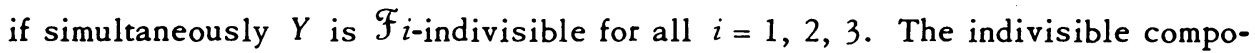
nents (cf. (2.5)) of a set $Y \subset X$ for the three kinds of indivisibility notions are

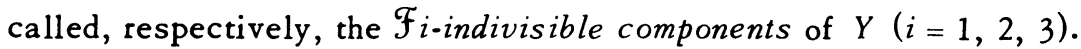

In [10] we consider indivisibilities only for the case when $N(X)$ is of 0 -dimension. The statement there that a set $Y \subset X$ is indivisible by $T$ is the same as $2_{0} X Y$ in the terminology of (3.1). The statement that $Y$ is $C C$-indivisible by $T$ is the same as that $2_{0} X Y$ and $2_{0} \| Y$ both hold.

3.2. Remark. Let $Y \subset X$ and $\mathcal{F} \subset$ 2. Then the following properties hold:

(1) If $\mathfrak{F} X Y$, then $\mathfrak{F} \not Y \Leftrightarrow \mathcal{F} \| Y$.

(2) If $\mathfrak{F} \| Y Y$, then $\mathfrak{F} X Y \Leftrightarrow \mathcal{F} \pitchfork Y$.

In case $Y$ is a connected subset of $E(X)$, a stronger version of (3.2) is given in (4.5).

The consideration of whether a set is $\mathfrak{F}_{3}$-indivisible is superfluous if one is only interested in transformation groups with compact phase spaces. However, even for cases when the phase spaces $X$ are locally compact (and noncompact) this condition is an important one. We will show later that in certain cases the completeness of $X$ can ensure this condition (3.12).

In order to analyze the three kinds of indivisibilities defined in (3.1) we need the following technical theorem. This theorem is responsible for the failure of many subsequent results on nonlocally compact spaces.

3.3. Theorem. Let $K$ be an invariant subset of $X, a \in \mathcal{Q}, u \in \mathcal{U}, x \in E(X)$ and $\left\{x_{i}\right\}$ a net in $X$, which satisfy the following properties:

(a) $x u^{4} \cap K=\varnothing$.

(b) $\mathrm{Cl}\left(x u^{3}\right)$ is compact.

(c) $\left\{x_{i}\right\}$ converges to $x$.

(d) For each $i$ the net $x_{i}$ a bas a limit point in $X-F_{u}(X)$ (cf. (1.2)). Then $x a$ bas a limit point in $X-K$.

Proof. Since $x \in E(X)$, there exists $v \in \mathcal{U}, v \subset u$, such that $y \in X,(x, y) \epsilon$ $v$ imply $(x t, y t) \in u$ for all $t \in T$. Without loss of generality we may assume that the points $\left\{x_{i}\right\}$ are all in $x v$. Fix a point, say $x_{1}$, in $\left\{x_{i}\right\}$. By replacing a with a subnet we may assume that $\lim x_{1} \alpha=y_{1} \in X-F_{u}(X)$. By the definition of $F_{u}(X)$ there exists $w \in \mathcal{U}$ such that

$$
z_{i} \in X(i=1,2), \quad\left(y_{1}, z_{i}\right) \in w \Rightarrow\left(z_{1} t, z_{2} t\right) \in u, \text { for all } t \in T .
$$

Let $a=\left\{t_{j}\right\}$. There exists some term $s=t_{j_{1}}$ of a such that $\left(y_{1}, x_{1} t_{j}\right) \in w$ for all $j \geq j_{1}$. For such $j$ we have 


$$
\left(x, x t_{j} s^{-1}\right)=\left(x, x_{1}\right)\left(x_{1} s s^{-1}, x_{1} t_{j} s^{-1}\right)\left(x_{1} t_{j} s^{-1}, x t_{j} s^{-1}\right) \in u^{3} .
$$

It follows from (b) that there is a subnet of $\left\{x t_{j} s^{-1}\right\}_{j}$ which converges to a point $p \in x u^{4}$. By (a) we have that $p \notin K$. Since $K$ is invariant, the net $x a=\left\{x t_{j}\right\}_{j}$ then has a subnet which converges to a point $q=p s \notin K$.

3.4. Corollary. Suppose $X$ is locally compact, $x \in E(X), \alpha \in \mathcal{2}$ and $\lim x \alpha=$ $p \notin E(X)$. Then there exists a neighborbood $U$ of $x$ and $u \in \mathcal{U}$ such that $y \beta$ bas a limit point in $F_{u}(X)$, for every $y \in U$ and for every subnet $\beta$ of $\alpha$.

Proof. Let $K=\mathrm{Cl}(p T)$. By Remark 1.6(1) we have that $K \subset N(X)$. We can then choose $u \in \mathcal{U}$ to satisfy conditions (a) and (b) of Theorem 3.3. By the local compactness at $p$ and that $x \in E(X)$ we have a compact neighborhood $W$ of $p$ and a neighborhood $V$ of $x$ such that $z \alpha$ is eventually in $W$ for all $z \in V$. The corollary then follows immediately from the theorem. The neighborhood $U$ turns out to be a subneighborhood of $V$.

3.5. Corollary. Let $X$ be locally compact, $a \in 2,\left\{x_{i}\right\}$ a net in $X$ which converges to $x \in E(X)$ and that every subnet of $x_{i}$ a bas a limit point in $E(X)$ for all $i$. Then every subnet of $x \alpha$ bas a limit point in $E(X)$.

Proof. By first letting $K$ be $F_{u}(X)$ for some small $u \in \mathcal{U}$, Theorem 3.3 shows that every subnet of $x a$ has at least one limit point. If one such limit point, say $p$, is not in $E(X)$. Then $p \in F_{v}(X)$ for some $v \in \mathcal{U}, v \subset u$. Now there exists a subnet $\beta$ of $\alpha$ such that $\lim x \beta=p$. By applying Theorem 3.3 again for $K=F_{v}(X)$ and with $\beta$ in place of $\alpha$ we obtain a contradiction.

In the previous two corollaries the set $K$ is a subset of $N(X)$. Though it is not as important for the sequel, dynamical results may be obtained by letting $K$ too be a subset of $E(X)$, as illustrated in the following.

3.6. Corollary. Let $X$ be locally compact, then every orbit closure which lies entirely in $E(X)$ is a minimal set. Moreover, the set of all minimal sets contained in $E(X)$ is relatively closed in $E(X)$.

Proof. First assertion. Let $\mathrm{Cl}(x T) \subset E(X)$ and assume that $\mathrm{Cl}(x T)$ is not minimal. Then there exists a closed nonempty invariant subset $K$ of $\mathrm{Cl}(x T)$ such that $x \notin K$. For $y \in K$ there is some $\alpha \in \mathcal{Q}$ such that $\lim x \alpha=y$. Let all $x_{i}$ in Theorem 3.3 be just $x$; then we have a contradiction. The first assertion does not really show the significance of the theorem, since a direct proof may be obtained without assuming that $X$ is locally compact. The 'moreover' part does require this local compactness. Here we may assume that the $x_{i}{ }^{\prime} s$ in the theorem lie in a minimal set $M_{i} \subset E(X)$. If $\lim x_{i}=x \in E(X)$, it would follow from Corollary 3.5 that $\mathrm{Cl}(x T) \subset E(X)$; hence the first assertion of the corollary applies. 
We now apply Theorem 3.3 to study the $\mathcal{F}_{1}$ - and $\mathcal{F}_{3}$-indivisible components of a subset of $E(X)$.

3.7. Lemma. Let $X$ be locally compact, $\mathcal{F}$ a hereditary family of 2 and $K=$ $\left\{\mathcal{K}_{j}\right\}$ a decomposition of $N(X)$ into compact sets. Let $Y \subset E(X)$ and $D$ be a dense subset of $Y$. Then $(\xi, Y, \mathcal{F}, \mathcal{K})$ is an indivisibility $\leftrightarrow(\xi, D, \mathcal{F}, \mathcal{K})$ is an indivi. sibility.

Proof. The $(\Rightarrow$ part follows from Proposition 2.3(2).

We prove $\Leftrightarrow$ by contradiction. Thus assume that for some $K=K_{j_{0}}$ there exists $\alpha \in \mathcal{F}$ such that $(\xi, Y, \alpha, K)$ is not an indivisibility. Let $\beta$ be the subnet given in (2.6). Then $\beta \in \mathcal{F}$ and $(\xi, Y, \beta, K)$ is not an indivisibility. There exist $y_{0}, y_{1} \in Y$ such that $\lim y_{0} \beta \in K$ and $y_{1} \beta$ has no limit point in $K$.

Case 1. $y_{0} \in D$. Since $(\xi, D, \beta, K)$ is an indivisibility, we have $\lim z \beta \in K$ for all $z \in D$. Since $C l(D) \supset Y$ and $y_{1} \in E(X), K$ compact, we must have $\lim y_{1} \beta \in K$, which gives a contradiction.

Case 2. $y_{0} \notin D$. By Corollary 3.5 there is some $z_{0} \in D$ such that $p=$ $\lim z_{0} \beta \notin E(X), p \in X$. Then there exists some $K^{\prime}=K_{j_{1}}$ such that $p \in K^{\prime}$. Applying the argument of the first case we have $K^{\prime}=K$ and then Case 2 is re. duced to Case 1. The proof of the lemma is complete.

The main application of (3.7) is for the case that $N(X)$ has compact components: then the set $\left\{K_{j}\right\}$ is the set of all components of $N(X)$.

A similar argument to the one given in (3.7) yields the following results.

3.8. Lemma. Let $X$ be locally compact, $\mathcal{F}$ a bereditary family of $2, Y \subset$ $E(X)$ and $D$ a dense subset of $Y$. Then

(1) If $\mathcal{F} \mathbb{K} Y$ and if $\mathcal{K}=\left\{K_{j}\right\}$ is a decomposition of $N(X)$ into closed subsets, then $(\xi, Y, \mathcal{F}, \mathcal{K})$ is an indivisibility $\Leftrightarrow(\xi, D, \mathcal{F}, \mathcal{K})$ is an indivisibility.

(2) If $K$ is compact, $K \subset N(X)$, and relatively open in $N(X)$, then $(\xi, Y, \mathcal{F}, K)$ is an indivisibility $\leftrightarrow(\xi, D, \mathcal{F}, K)$ is an indivisibility.

3.9. Theorem. Suppose $X$ is locally compact, $\mathcal{F} \subset \mathcal{Q}$ is bereditary, $Y \subset E(X)$ and either the condition

(a) $N(X)$ bas compact components

or

(b) $\mathcal{F} \cdot \|$ Y $Y$ and $N(X)$ bas closed components

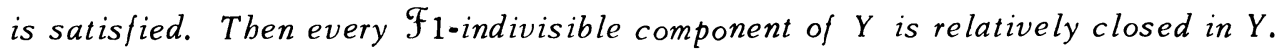

Proof. Use (3.7) and (3.8(1)).

The approach to third-indivisibility is different from that of first-indivisibility.

3.10. Remark. Let $X$ be locally compact, $\mathcal{F}$ a hereditary family in $2, Y \subset X$ and let $\xi^{*}=\left(X^{*}, T, \pi^{*}\right)$ be the extended transformation group as given in (2.10). 
Then $\mathcal{F} \| Y \Leftrightarrow\left(\xi^{*}, Y, \mathcal{F} ;\{\infty\}\right)$ is an indivisibility.

Proof. Use (2.8).

3.11. Theorem. Let $X$ be locally compact, $\mathcal{F} \subset \mathcal{Q}$ and $Y \subset E(X)$. Then the following properties bold:

(1) If $\mathcal{F}$ is a singleton which is a net converging pointwise in $X$ to $X^{*}$ and $X$ is complete, then there are exactly one or two $\mathfrak{F}_{3}$-indivisible components of $Y$ and they are relatively open and closed in $Y$.

(2) If $X$ is complete and $\mathcal{F}$ is bereditary, then every $\mathfrak{F}_{3}$-indivisible component of $Y$ is relatively closed in $Y$.

(3) If $X$ is uniformly locally compact (i.e. there exists $u \in \mathcal{U}$ such that $x u$ is compact for every $x \in X)$ [8, p. 214], [1, p. 211], then every $\mathfrak{F}_{3 \text {-indivisible }}$ component of $Y$ is relatively open and closed in $Y$.

Proof. Statement (1). Let $\mathcal{F}=\{a\}$. Let $A=\{y \in Y \mid \lim y a=\infty\}$ and $B=$ $\{y \in Y \mid \lim y \alpha \in X\}$. Clearly then $A$ and $B$ are the only two $\mathfrak{F}_{3}$-indivisible components of $Y$. It follows from (2.11) that $A$ is relatively closed in $Y$. The relative openness of $A$ follows from the completeness of $X$.

Statement (2) follows from statement (1), (2.8) and (2.4(3)).

Statement (3) can be obtained easily by arguing on $Y \subset E(X)$. Thus let $u \in \mathcal{U}$ such that $x u$ is compact for every $x \in X$. Let $v \in \mathcal{U}, v^{3} \subset u$. Let $I$ be any $\mathfrak{F}_{3}$ indivisible component of $Y$ and let $a \in \mathrm{Cl}(I) \cap Y$. Since $a \in E(X)$, there exists $w \in \mathcal{U}$ such that

$$
\left(a, y_{i}\right) \in w(i=1,2) \Rightarrow\left(y_{1} t, y_{2} t\right) \in v, \text { for all } t \in T .
$$

By (2.3(3)) it suffices to show that $\mathcal{F} \|$ aw. Suppose $\mathcal{F} \| a w$, then there exist $y_{1}, y_{2} \in a w$ and $a \in \mathcal{F}$ such that $y_{1} a$ has a limit point $p \in X$ and $y_{2} \alpha$ has no limit point in $X$. By the choice of $w$, however, $y_{2}$ a must have a limit point in $p u$, which is a contradiction. The proof of statement (3) is complete.

3.12. Corollary. Let $X$ be complete and locally compact $\mathcal{F} \subset \mathcal{Q}$ bereditary, $Y \subset E(X), Y$ connected; then $\mathcal{F} \| Y$.

Proof. Use (2.8) and (3.11(1)).

By using the stronger condition that $X$ is uniformly locally compact instead of completeness in (3.12), the requirement that $\mathcal{F}$ be hereditary can be omitted. This fact can be obtained from (3.11(3)).

4. Topological sufficient conditions for indivisibility. Let $(\xi, Y, \mathcal{F}, \mathcal{K})$ be a $C$-object. In this section we seek topological conditions on $X, Y$ and members of $\mathcal{K}$ which guarantee that $(\xi, Y, \mathcal{F}, \mathcal{K})$ is an indivisibility, or equivalently that the set $Y$ is its own indivisible component. We will confine the set $Y$ to being a 
subset of $E(X)$ and will mainly be concerned with the complete indivisibility property. Results which exceed complete indivisibility are also obtained (cf. (4.11)). It is also possible to apply our results to the case when $Y \not \subset E(X)$ by considering a suitable decomposition of $X$ so that the projection of $Y$ in the quotient transformation group consists of equicontinuous points only. We will, however, not discuss such a generalization in this paper.

We first note the following two important remarks. First, if the set $Y$ is disconnected, a criterion can hardly be obtained. Hence it is natural to restrict the set $Y$ to being connected, or equivalently to find conditions for a set $Y \subset E(X)$ so that the indivisible components of $Y$ are larger than the topological components. Second, topological sufficient conditions for the case $\mathcal{F}=\mathcal{Q}$ are most desirable since if $(\xi, Y, \mathcal{Q}, \mathcal{K})$ is an indivisibility then $(\xi, Y, \mathcal{F}, \mathcal{K})$ is an indivisibility (cf. (2.3(5))).

Throughout the remainder of this paper, unless explicitly stated otherwise, we will make the standing bypothes is that $X$ is locally compact (uniform $T_{2}$ ) and that $Y$ is a connected subset of $E(X)$.

We now state the two main theorems of this section. Recall that the statement " $Y$ is completely indivisible by 2 " has been denoted by $2 \pitchfork Y$.

4.1. Theorem. If $2 \| Y$ (such is the case if $X$ is complete), then every one of the following conditions implies that $2 \pitchfork Y$ :

(1) $Y \cap \mathrm{Cl}(N(X))=\varnothing$.

(2) $Y$ is locally compact invariant.

(3) $N(X)$ is scattered.

Moreover, if $N(X)$ is 0-dimensional( $\left.{ }^{1}\right)$ (every $p \in N(X)$ bas arbitrarily small, both relatively open and closed neighborboods), then the bypothesis $2 \mathbb{Y} Y$ is not needed for (1), (2), and (3).

4.2. Theorem. If the components of $N(X)$ are compact, then every one of the following conditions implies that $2 \pitchfork Y$ :

(1) $Y$ is a semicontinuum.

(2) Either $X$ or $Y$ is locally connected.

(3) $N(X)$ is locally compact.

In case $N(X)$ has compact components, which is already assumed in (4.2), then according to (3.9) the set $Y$ in all of the conditions in the two theorems can be replaced by a dense subset of it.

Proofs of the statements in Theorems 4.1 and 4.2 will be given separately, together with other interesting results.

(1) This condition can equally well be replaced by the condition that $\mathrm{Cl}(N(X))$ is compact (cf. remark following (4.14)). 
In order to demonstrate the necessity of splitting the sufficient condition for $2 \pitchfork Y$ into various cases as given in (4.1) and (4.2), we give two simple examples which show that the connectedness of $Y$ alone does not give $2 \pitchfork Y$.

4.3. Example. Let $b$ be a self-homeomorphism of the unit circle $C$ which has neither periodic points nor dense orbits. The discrete flow on $C$ generated by $b$ then has a unique minimal set $M$ which is homeomorphic to the Cantor set (cf. for example [12]). If $I$ is a complementary interval of $M$, then $I \cap\left(I b^{n}\right)=\varnothing$ for all integers $n \neq 0$. Since $C$ has finite measure, we conclude that $\lim _{|n| \rightarrow \infty}$ diameter $\left(I b^{n}\right)=0$. It follows that $I \subset E(C)$. By Remark $(1.6(2))$ we have that either $M \subset E(C)$ or $M \subset N(C)$. If $M \subset E(C)$ then $C=E(C)$ and it is well known that in this case $b$ is topologically conjugate to either a rotation or to a reflection with respect to the $x$-axis. The assumption for $b$ would then be violated. Hence $N(C)=M$. Now we let $X$ be the closed unit disk. We extend $b$ to $X$ be requiring that each segment which joins the origin 0 to a point $z \in C$ be rotated around 0 so that $z$ is mapped to $z h$. Let $M^{\prime}$ be the union of all segments joining 0 to points in $M$. It is clear that $N(X)=M^{\prime}-\{0\}$. Let $Y$ be the union of all segments joining 0 to a complementary interval $I$ of $M$ in $C$. The set $Y$ is connected and, in fact, arcwise connected; but it is clear then $2 \mid Y$. In fact it is not difficult to see that $\{0\}$ and $Y-\{0\}$ are the two 21-indivisible components of $Y$. Theorem 4.2 does not apply, since the components of $N(X)$ are not compact. Theorem 4.1 does not apply, since neither one of the conditions is satisfied.

4.4. Example. Let $I$ be the unit interval $[0,1]$ and consider a continuous flow on $I$ whose only fixed points are $\{0,1\}$. The flow is extended naturally to the unit square $I \times I$ by requiring that points on every vertical line be translated horizontally as its projection on the $x$-axis. Let $X=I \times I-\{p\}$ where $p$ is the point $(0,1 / 2)$ in the plane. Let $J$ be the interior interval of $I$. It is clear that $E(X)=J \times J$. Let $Y=E(X)$. It is not difficult to see that $2 \mid Y$. In fact there are three 21-indivisible components of $Y$. They are the intersection $A$ of $Y$ with the line $y=1 / 2$ and the two complementary domains of $A$ in $Y$. Theorem 4.1 does not apply, since $2 \| \mid Y$. Theorem 4.2 does not apply, since two of the three components of $N(X)$ are not compact.

Example 4.3 can easily be extended to the 2-sphere by piecing up two disks. Example 4.4 can be modified to obtain a similar one on the two-dimensional torus with a point punctured (add the reflection of $X$ with respect to the $y$-axis and identify parallel edges of the resulting rectangle).

We now begin our topological analysis. The three kinds of indivisibility defined in (3.1) are related as shown in (3.2). For a connected set $Y \subset E(X)$ a stronger relation can be obtained.

4.5. Proposition. If $\mathcal{F}$ is a bereditary family of 2 , then the following properties bold: 
(1) $\mathfrak{F} \backslash Y \leftrightarrow \mathscr{F} \pitchfork Y$.

(2) If $Y$ is an arbitrary subset of $E(X)$ such that $2 \| Y$ (sucb is the case if $Y$ is connected and $X$ is complete), then

$$
\mathfrak{F} X y \Leftrightarrow \mathcal{F} \forall Y \leftrightarrow F) \pitchfork Y .
$$

Proof. By (3.2(1)), to show statement (1) it suffices to prove that $\mathcal{F} X Y \Rightarrow$ $\mathcal{F} \| Y$. We prove it by contradiction. Thus assume that there exists $\alpha \in \mathcal{F}$ and $a \| Y$. Let $\beta$ be the subnet of $\alpha$ given in (2.6). Then $\beta \in \mathcal{F}$ and $\beta \| Y$. We consider the following nonempty set:

$$
A=\{x \in Y \mid \lim x \beta=\infty\},
$$

where $\infty$ is the point at infinity of the one-point compactification of $X$. It follows from (2.11) that the set $A$ is relatively closed in $Y$. By (3.5) every $a \in A$ has a neighborhood $V_{a}$ such that if $x \in V_{a}$, then $\lim x \beta \notin E(X)$. Since $\mathcal{F} X Y$ and $\beta \epsilon$ $\mathcal{F}$, we must have that $V_{a} \cap Y \subset A$. Hence $\Lambda$ is also relatively open in $Y$. Since $Y$ is connected, we have $A=Y$ and therefore $\beta \| Y$. We then have a contradiction.

Statement (2). It suffices to show that $\mathfrak{F} \not Y Y \Rightarrow F \backslash Y$. We again use a proof by contradiction. Let $\beta \in \mathcal{F}$ be the net given in (2.6) such that $\beta \mid Y$. Since $\beta \| Y$, we have that $\lim y \beta \in X$ for every $y \in Y$. The function $f: Y \rightarrow X$ defined by $y f=\lim y \beta$ is easily seen to be continuous. Since $\beta \mid Y$, there exists $y_{0} \in Y$ such that $y_{0} f \in N(X)$. Since $\beta \| Y$, we must have that $y f \in N(X)$ for every $y \in Y$. The set $Y f$ is then a connected subset of $N(X)$, whence it lies entirely in a component of $N(X)$. This contradicts that $\beta \mid Y$,

The proof of the proposition is complete.

We now prove Theorem 4.1.

4.6. Lemma. If $2 \mathbb{Y} Y$ and $2 \mid Y$, then there exist $\beta \in$ 2, $a \in Y \cap \operatorname{Cl}(N(X))$ and a net $\left\{x_{i}\right\}$ in $Y$ which satisfy the following properties:

(1) $\lim x \beta \in X$ exists, for all $x \in Y$.

(2) $\lim x_{i}=a$.

(3) $\lim x_{i} \beta \in N(X)$, for all i.

Proof. Let $\beta \in \mathcal{2}$ be the net given in (2.6) for $2 \mid Y$. Then $\beta \mid Y$. Since $2 \| Y$, we have that $\lim x \beta \neq \infty$ for all $x \in Y$, which shows statement (1). Note that (2.6) permits us to replace $\beta$ by a subnet so that in addition we also have that $\lim x \beta^{-1} \in X^{*}$ exists for all $x \in X$. By (3.5) the set $A=\{x \in Y \mid \lim x \beta \epsilon$ $E(X)\}$ is relatively closed in $Y$. The set $A$ is not relatively open in $Y$, due to the fact that $Y$ is connected and $\beta \mid Y$. Hence there exist $a \in A$ and a net $\left\{x_{i}\right\} \subset Y$ - $\Lambda$ such that $\lim x_{i}=a$. Since $\lim x_{i} \beta \in N(X)$ for all $i$, we have that $b=: \lim a \beta$ $\epsilon E(X) \cap \mathrm{Cl}(N(X))$. Then $\lim b \beta^{-1}:: a$. Note that the set $\mathrm{Cl}(N(X))$ is closed and invariant. Hence a $\in Y \cap \mathrm{Cl}(N(X))$. The proof of the lemma is complete. 
4.7. Proof of Theorem 4.1(1). Use (4.5) and (4.6).

4.8. Proof of Theorem 4.1(2). It suffices to show that $2 X Y$ (use (4.5)). We need the fact that every locally compact subset of a topological space is relatively open in its closure [9, Vol. 1, p. 65]. The set $\mathrm{Cl}(Y)-Y$ is then closed and invariant. Assume now $2 \mid Y$. Using the same notation as that given in the proof of (4.6), we observe that the point $b(=\lim a \beta)$ is not in $Y$, or $Y$ would fail to be locally compact at $b$. Hence $b \in \mathrm{Cl}(Y)-Y$. But then $a=\lim b \beta^{-1} \in \mathrm{Cl}(Y)-Y$. We have a contradiction, and the proof is complete.

Most of the results in this work can actually be obtained under some weaker hypotheses. For instance, those components of $N(X)$ which are disjoint from $\mathrm{Cl}(y T)$ for all $y \in Y, Y \subset E(X)$, have nothing to so with the 21 -indivisibility for $Y$. Restrictions imposed on these components are therefore superfluous and, on occasion, also obstacles to obtaining results.

Let $\sigma=(\xi, Y, \mathcal{F}, \mathcal{K})$ be a $C$-object. For the moment we do not assume $Y \subset$ $E(X)$. For $x \in X$ and $\alpha \in \mathcal{F}$ we denote $\overline{\lim } x \alpha$ to be the set of all limit points of the net $x a$. We now introduce a notion of accessibility.

4.9. Definition. The $\sigma$-accessible subfamily of $\mathcal{K}$ is defined to be the family

$$
\mathcal{K}_{\sigma}=\{K \in \mathcal{K} \mid K \cap[\bigcup\{\overline{\operatorname{Iim}} y a \mid y \in Y, a \in \mathcal{F}\}] \neq \varnothing\} \text {. }
$$

We will also say a $K \in \mathcal{K}_{\sigma}$ is accessible by $(Y, \mathfrak{F})$.

The following statement is obvious.

4.10. Remark. Let $\sigma=(\xi, Y, \mathcal{F}, \mathcal{K})$ be a $C$-object. Then $\sigma$ is an indivisibility $\Leftrightarrow\left(\xi, Y, \mathcal{F}, \mathcal{K}_{\sigma}\right)$ is an indivisibility.

For a subfamily $\mathcal{K} \mathcal{K}$, by $S(£)$ we will denote the subset of the space $X$, which is the union of all members of $\mathscr{L}$. If members of $\mathcal{L}$ are pairwise disjoint we will make $\mathcal{L}$ a topological space by giving it the quotient topology of $S(£)$.

We recall some terminology and results of general topology. A topological space is said to be scattered if it contains no self-dense subset (cf. [9, Vol. 1, p. 78]). Every $T_{1}$ scattered space is necessarily totally disconnected. If in addition the space has a countable base, then it is at most countable [13, p. 63]. Every topological space $S$ can be uniquely expressed as the disjoint union of a perfect set $P$ and a scattered set [9, Vol. 1, p. 79]. The perfect set $P$, which is in fact the union of all self-dense subsets of $S$, is called the kernel of $S$ in Kuratowski [9] and the nucleus of $S$ in Sierpiński [13, p. 13]. Every discrete space is clearly scattered. Examples of nondiscrete scattered spaces include the ordinal spaces and many others (cf. [14]).

We now restrict $\mathcal{K}$ to be a decomposition of $N(X)$ and resume the hypothesis that $Y \subset E(X), Y$ connected. 
4.11. Theorem. Let $\sigma=(\xi, Y, \mathcal{F}, \mathcal{K})$, where $Y \subset E(X), Y$ connected, $\mathcal{F} \subset \mathcal{Q}$ bereditary, and $\mathcal{K}$ a decomposition of $N(X)$ into compact subsets. Then the follow. ing properties bold:

(1) If $\mathcal{L} \subset \mathcal{K}_{\sigma}, S(\mathcal{Q})$ is relatively open in $S\left(\mathcal{K}_{\sigma}\right)$ and $\mathcal{L}$ is scattered, then $(\xi, Y, \mathfrak{F}, \mathfrak{Q})$ is an indivisibility.

(2) If $\mathcal{K}_{\sigma}$ is scattered, then $\sigma$ is an indivisibility.

(3) Let $\mathscr{P}$ denote the kernel of $\mathcal{K}_{\sigma}$. Then $\sigma$ is an indivisibility $\leftrightarrow(\xi, Y$, $\mathfrak{F}, \mathfrak{P})$ is an indivisibility.

Proof. Statement (1). We show that if $(\xi, Y, \mathcal{F}, \mathfrak{\complement})$ is not an indivisibility, then $\mathfrak{L}$ contains a self-dense subset. Thus assume that for some $a \in \mathcal{F}$ the 4 tuple $(\xi, Y, \alpha, \mathfrak{Q})$ is not an indivisibility. Let $\beta$ be the subnet of $\alpha$ given in (2.6). Consider the nonempty family

$$
\mathscr{L}_{\beta}=\left\{K \in \mathscr{L} \mid K \cap\left[\bigcup_{y \in Y} \lim y \beta\right] \neq \varnothing\right\}
$$

(that $\mathscr{Q}_{\beta} \neq \varnothing$ is due to the assumption that $(\xi, Y, \beta, \mathfrak{Q})$ is not an indivisibility). We assert that $\mathscr{L}_{\beta}$ is self-dense. Suppose the contrary; then the space $\mathscr{L}_{\beta}$ has an isolated point $L_{0}$. Then $L_{0}$, regarded as a set in $X$, is relatively open in $s\left(\varrho_{\beta}\right)$. Now consider the family

$$
\mathcal{K}_{\beta}=\left\{K \in \mathcal{K}_{\sigma} \mid K \cap\left[\bigcup_{y \in Y} \lim y \beta\right] \neq \varnothing\right\} .
$$

Using the hypothesis that $S\left(\mathcal{L}^{\mathcal{D}}\right)$ is relatively open in $S\left(\mathcal{K}_{\sigma}\right)$, it is not difficult to show that $S\left(\complement_{\beta}\right)$ is relatively open in $S\left(\mathcal{K}_{\beta}\right)$. In fact we have that $S\left(£_{\beta}\right)=S(\varrho) \cap$ $s\left(\mathcal{K}_{\beta}\right)$. Hence $L_{0}$ is relatively open in $S\left(\mathcal{K}_{\beta}\right)$. Now let $y_{0} \in Y$ such that $\lim y_{0} \beta \in L_{0}$. It follows from the compactness of $L_{0}$ that the indivisible component $I_{y_{0}}=I_{y_{0}}\left(\xi, Y, \beta, L_{0}\right)$ is relatively closed in $Y$. By using (3.5) and the fact that $L_{0}$ is relatively open in $S\left(\mathcal{K}_{\beta}\right)$, it is not difficult to see that every $y \in I_{y_{0}}$ has a relative neighborhood $V(y)$ in $Y$ such that if $z \in V(y)$ then $\lim z \beta \in L_{0}$. Hence $I_{y_{0}}$ is also relatively open in $Y$. Since $Y$ is connected, we conclude that $Y=I_{y_{0}}$. Then $\lim y \beta \in L_{0}$ for all $y \in Y$. The last statement contradicts that $(\xi, Y, \beta, \mathcal{L})$ is not an indivisibility. Hence $\mathcal{L}_{\beta}$ is self-dense and statement (1) is proved.

Statement (2) follows from statement (1) and (4.10).

Statement $(3)$. The $(\Rightarrow)$ part follows from $(2.3(6))$. For the $(\Leftarrow)$ part we note that the family $\mathcal{K}_{\sigma}-\mathcal{P}$ is scattered and relatively open in $\mathcal{K}_{\sigma}$. Hence $s\left(\mathcal{K}_{\sigma}-\mathcal{P}\right)$ is relatively open in $S\left(\mathcal{K}_{\sigma}\right)$. By statement (1) we have that $(\xi, Y, \mathcal{F}$, $\left.\mathcal{K}_{\sigma}-\mathcal{P}\right)$ is an indivisibility. Since we also assume that $(\xi, Y, \mathcal{F}, \mathcal{P})$ is an indivisibility, we have that $\left(\xi, Y, \mathcal{F}, \mathcal{K}_{\sigma}\right)$, whence $\sigma$ itself is an indivisibility.

The proof of the theorem is complete.

We note that the compactness assumption for members of $\mathcal{K}$ in (4.11) is essential (take $\mathcal{F}=\mathcal{2}$ and $\mathcal{K}$ be the three components of $N(X)$ in (4.4)). 
We now apply Theorem 4.11 to complete indivisibility.

4.12. Theorem. Suppose $K_{\sigma}$, the set of all components of $N(X)$ accessible by $(Y, 2)$, has compact members and that it is scattered (under the quotient topology of $\left.S\left(\mathcal{K}_{\sigma}\right)\right)$ : then $2 \pitchfork Y$.

4.13. Proof of Theorem 4.1(3). Since $N(X)$ is scattered, it is totally disconnected. The components of $N(X)$ are then points of $N(X)$. If $\mathcal{K}$ denotes the set of all components of $N(X)$ with quotient topology, then $K$ is homeomorphic to $N(X)$. Then $\mathcal{K}$, whence $\mathcal{K}_{\sigma}$, is scattered and we can apply (4.12).

We note that if $X$ has a countable basis, then (4.13) actually concerns only transformation groups such that $N(X)$ is countable. However, we will see in the next section that the scatteredness condition cannot be replaced by countability (5.4).

4.14. Proof of the last statement of Theorem 4.1. The key for the proof is using one-point compactification. First by (2.11) we have that $Y \subset E\left(X^{*}\right)$ and $N\left(X^{*}\right) \subset\{\infty\} \cup N(X)$. Since $N(X)$ is 0 -dimensional, it is easily verified that the set $\{\infty\} \cup N(X)$, whence the set $N\left(X^{*}\right)$, is totally disconnected. We must show 2 $\| Y Y$ in the transformation group $(X, T, \pi)$. Suppose not; then $\infty \in N\left(X^{*}\right)$. We now apply the first three statements to the $C$-object $\left(\xi^{*}, Y, 2, \mathcal{K}\right)$, where $\xi^{*}=$ $\left(X^{*}, T, \pi^{*}\right)$ and $\mathcal{K}$ is now the set of components of $N\left(X^{*}\right)$. In particular, as a result that $\left(\xi^{*}, Y, 2,\{\infty\}\right)$ is an indivisibility implies that $(\xi, Y, 2,\{X\})$ is an indivisibility, or equivalently $2 \| Y$ (cf. (3.10)), which gives a contradiction.

The last statement of Theorem 4.1 can clearly be generalized in various ways, since $N(X)$ was assumed to be 0 -dimensional only in order to have $\{\infty\}$ as a component of $N\left(X^{*}\right)$.

In order to prove Theorem 4.2(1) we will need the following theorem which can be found in the standard literature of general topology.

4.15. Theorem. Let $S$ be a $T_{2}$ continuum, let $U$ be any open nonempty proper subset of $S$ and let $C$ be a component of $U$. Then $\bar{U}-U$ contains a limit point of $C$.

We recall that a topological space is said to be a semicontinuum if every pair of its points can be joined by a continuum (cf. [9, Vol. 2, p. 188]).

4.16. Proof of Theorem 4.2(1). According to (2.3(3)) and (2.4(1)) it suffices to show for this special case that $Y$ is a continuum in $E(X)$. By $(4.5(1))$ we can reduce the problem to considering $2 X Y(Y$ a continuum in $E(X))$. We prove the latter by contradiction. Thus assume that $\alpha \mid Y$ for some $\alpha \in 2$. Let $\beta$ be the subnet of $\alpha$ given in (2.6). Then $\gamma \mid Y$ for all subnets $\gamma$ of $\beta$. Consider the following sets: 


$$
\begin{aligned}
& A=\{y \in Y \mid \lim y \beta \in E(X)\}, \quad B=\{y \in Y \mid \lim y \beta=\infty\}, \\
& F=A \cup B, \quad U=Y-F=\{y \in Y \mid \lim y \beta \in N(X)\} .
\end{aligned}
$$

Note that $A$ is closed by (3.5) and $B$ is clearly closed; hence $F$ is closed. Since $\beta \mid Y$, we have $U \neq \varnothing$. Consider the continuous map $f: Y \rightarrow X^{*}=X \cup\{\infty\}$ given by $y f=\lim y \beta, y \in Y$. If $F=\varnothing$, then $Y f=U f \subset N(X)$ and we would have that $\beta X Y$, by the connectedness of $Y f$. Hence we also have $F \neq \varnothing$ and so (4.15) applies. Let $C$ be a component of $U$. Then $C f \subset N(X)$; hence there is a component $K$ of $N(X)$ such that $\lim y \beta \in K$ for all $y \in C$. We now argue in the terminology of (2.4). This will make the proof slightly longer, but more transparent, since it will show the advantage of having closed indivisible components. We let $\mathfrak{F}$ denote the class of all subnets of $\beta$. Then $C \subset I_{c}(\xi, Y, \mathcal{F}, K)$, where $c$ is a point of $C$. By (4.15) there exists some $z \in F \cap \mathrm{Cl}(C)$. By (3.9) we have that $z \in$ $I_{c}(\xi, Y, \mathcal{F}, K)$, which is impossible since we also have $z \in F=A \cup B$. We then have a contradiction and the proof is complete.

The previous example given in (4.4) shows that Theorem 4.2(1) is false if the condition that $N(X)$ have compact components is replaced by the condition that $N(X)$ have closed components.

4.17. Proof of Theorem 4.2(2). Suppose 2|Y. By applying (2.7), (3.5) and the local connectedness of $X$ or $Y$ we obtain easily that a certain 21-indivisible component $I$ of $Y$ is relatively open in $Y$. The set $I$ is relatively closed in $Y$ by (3.9). Since $Y$ is connected, we have a contradiction.

4.18. Remark. (1) Clearly the connectedness and local connectedness conditions on $Y$ in (4.2(2)) can be replaced by the assumption that every two points of $Y$ can be joined by a set which has such properties.

(2) The relative distance (due to Mazurkiewicz) between two points $x$ and $y$ of a metric space $S$ is defined to be

$$
\begin{aligned}
& \rho_{r}(x, y)=\inf \{\text { diameter } A \mid A \text { any connected set joining } x \text { to } y\} \\
& (\text { inf } \varnothing=-\infty) \quad[9, \text { Vol. 2, p. 250]. }
\end{aligned}
$$

In case $\rho_{r}$ is finite for all pairs, then it defines a metric on $S$. The new induced topology is usually stronger, but it coincides with the original one if $S$ is connected and locally connected to begin with. The following theorem can be shown by arguments similar to the proof in (4.17). If $X$ is a locally compact metric space and $N(X)$ has compact components and if a set $Y \subset E(X)$ has a finite $\rho_{r}$ and is connected under the new induced topology, then $2 \pitchfork Y$.

4.19. Proof of Theorem 4.2(3). It follows from the compactness of components of $N(X)$ and the local compactness of $N(X)$ that every component $K$ of $N(X)$ has an arbitrarily small, relatively open and compact neighborhood $W(K)$ in $N(X)$. Now suppose $2 \mid Y$. We let $\beta$ be the net given in (2.6) and let $I$ be a $\beta 1$-indivisible 
component of $Y$ such that $\lim y \beta \in K$ for all $y \in I$, where $K$ is a component of $N(X)$. For each fixed $W(K)$ it follows from (3.5) that the set $A=\{y \in Y \mid \lim y \beta \epsilon$ $W(K)\}$ is relatively open in $Y$. The set is clearly relatively closed in $Y$. Hence $A=Y$. It follows that $Y=I$ and this contradicts that $\beta \mid Y$. The proof is complete.

The proofs of Theorems 4.1 and 4.2 are now complete.

There is one more interesting result which is not covered by Theorems 4.1 and 4.2.

4.20. Theorem. If $2 \| Y$ ( $\operatorname{such}$ is the case if $X$ is complete), $N(X)$ bas compact components, $Y$ is locally compact and if $Y \cap \mathrm{Cl}(N(X))$ consists of fixed points, then $2 \pitchfork Y$. If $N(X)$ is 0-dimensional, the bypothesis $2 \| Y$ can be omitted.

Proof. The proof will depend heavily on the results of (4.1) and (4.2). In general, let $\mathrm{Cl}^{*}(A)$ denote the closure of a set $A \subset X$ taken in $X^{*}=X \cup\{\infty\}$. We consider the compact connected set $F=\mathrm{Cl}^{*}(Y)$ and let $G=F \cap[E(X)-$ $\mathrm{Cl}(N(X))]$. The set $G$ is then relatively open in $F$. Clearly we have the following inclusions:

$$
Y-\mathrm{Cl}(N(X)) \subset G \subset E(X) \cap \mathrm{Cl}^{*}[Y-\mathrm{Cl}(N(X))] .
$$

If $G=\varnothing$, then $Y \subset \mathrm{Cl}(N(X))$, whence $Y$ consists of fixed points and for this case $2 \pitchfork Y$. If $F-G=\varnothing$, then $F=G \subset E(X)-\mathrm{Cl}(N(X))$, whence by (4.1(2)) we also have $2 \pitchfork Y$. Without loss of generality we assume that $G \neq \varnothing$ and $F-G \neq \varnothing$. By (4.15) every component of $G$ has a limit point in $F-G \subset C l(N(X)) \cup\{\infty\}$. Assuming now $2 \mid Y$, we seek a contradiction. We can apply Lemma 4.6 and use the same notation. By the present hypothesis the point $a \in Y \cap \mathrm{Cl}(N(X))$ is a fixed point. Also the net $\left\{x_{i}\right\}$ is in $Y-\mathrm{Cl}(N(X))$, otherwise $\lim x_{i} \beta=x_{i} \in E(X)$. Let $G_{i}$ be the component of $G$ which contains $x_{i}$. By $(4.1(2))$ we have that $2 \chi G_{i}$. Hence $\lim y \beta=\lim x_{i} \beta=p_{i} \in N(X)$ for all $y \in G_{i}$ : Let $z_{i}$ be a limit point of $G_{i}$ in $F-G$. Then $z_{i} \notin Y$. For if $z_{i} \in Y$, then by (3.9) we have $2 \chi\left(G_{i} \cup\left\{z_{i}\right\}\right)$ and so $\lim z_{i} \beta=p_{i}$. On the other hand, if $z_{i} \in Y$, then $z_{i} \in Y \cap \mathrm{Cl}(N(X))$, which implies that $z_{i}$ is a fixed point, and then $\lim z_{i} \beta \neq p_{i}$, a contradiction. Now by the equicontinuity at $a$ we have $\lim p_{i}=\lim a \beta=a$ and it follows easily that $\lim z_{i}=a$. Since $z_{i} \notin \mathrm{Cl}^{*}[Y-\mathrm{Cl}(N(X))]-Y$, we have that $Y$ fails to be locally compact at the point $a$. We then have a contradiction. The proof of the first statement of the theorem is complete. The second statement follows from an argument similar to that given in (4.14).

In $[10$, Theorem 3$]$ we announce the following result.

4.21. Theorem. Let $X$ be a locally compact metric space. If $N(X)$ is 0 -di. mensional, then $E(X)$ is completely indivisible by $\mathscr{2}_{0}$ if it has a dense subset $D$ which satisfies one of the following conditions: 
(I) $D$ is a semicontinuum.

(II) $D$ is locally compact, connected and invariant (the condition "invariant" can be omitted if $T$ is connected).

(III) $D$ is connected and locally connected.

(IV) $D$ is connected and $N(X)$ is a discrete space.

(V) $X$ is uniformly locally compact and $D$ is the union of a family $\left\{M_{\lambda}\right\}$ of connected sets, which are totally ordered by inclusions and which satisfy $d\left(M_{\lambda}, N(X)\right)>0$ for all $\lambda$, where $d$ is the metric on $X$.

The previous theorem is a simple consequence of Theorems 4.1, 4.2, and 4.20. Theorem 4.20 is needed only for the parenthetical statement of (II). Note that for a connected group every component of an invariant set is also invariant. The set $N(X)$ is now totally disconnected, whence under connected group action it consists of fixed points. Since the fixed points of $X$ form a closed set, we see that $\mathrm{Cl}(N(X))$ consists of fixed points, hence (4.20) applies. That $E(X)$ can be replaced by a dense subset $D$ is already explained immediately following the statement of Theorem 4.2. Condition (IV) is now strengthened from that of a discrete set to a scattered set (4.1(3)). Condition (V) is also strengthened by (4.1(1)). $((2.3(4))$ is also needed for this proof.)

We now state the strengthened theorem in terms of uniform spaces.

4.22. Theorem. Let $X$ be a locally compact $T_{2}$ uniform space. If $N(X)$ is 0-dimensional, then $E(X)$ is completely indivisible by 2 if it has a dense subset $D$ which satisfies one of the following conditions:

(I) $D$ is a semicontinuum.

(II) D is locally compact, connected and invariant (the condition "invariant" can be omitted if $T$ is connected).

(III) $D$ is connected and either $D$ or $X$ is locally connected.

(IV) $D$ is connected and $N(X)$ is either scattered or locally compact.

(V) $D$ is the union of a family $\left\{M_{\lambda}\right\}$ of connected sets, which are totally ordered by inclusions and which satisfy $M_{\lambda} \cap \mathrm{Cl}(N(X))=\varnothing$ for all $\lambda$.

5. Concerning two fundamental questions. Let $X$ be, say, a compact connected metric space. The theorem of Homma-Kinoshita [6] says that if $N(X)$ is finite and does not disconnect the space $X$, then $N(X)$ consists of at most two points. A natural question is whether or not the previous statement still holds if the finiteness of $N(X)$ is replaced by countability.

Question 1. Does there exist a compact connected metric space $X$ whose $N(X)$ is countably infinite and does not disconnect the space? (This question arose during a conversation with Professor S. Kinoshita concerning the main theorem that he proved in [6].) 
This question is related to complete indivisibility in the following way. If one of the conditions given in Theorem 4.22 is satisfied, then $2 \pitchfork E(X)$ and according to [10, Theorem 2] we have that $N(X)$ has to be either a Cantor set or it consists of at most two points. For such cases the answer for Question 1 is negative. More generally, by Proposition 2.3(4), if the set $D$ in (4.22) is a transfinite monotone union consisting of semicontinua, sets which are both connected and locally connected, connected sets disjoint from $\mathrm{Cl}(N(X))$ and locally compact connected invariant sets, the answer for the question is negative.

$A$ related and perhaps more fundamental question is whether the conditions given in (4.22) can simply be replaced by the single condition that $E(X)$ be connected. More generally one may consider whether the six conditions given in Theorems 4.1 and 4.2 are essential enough, or even whether they can be omitted completely.

Question 2. If $X$ is a compact metric space and $N(X)$ has compact components, must every connected subset of $E(X)$ be completely indivisible by 2?

The main result of this section is to show by counterexamples that the answer for Question 1 is affirmative and that the answer for Question 2 is negative.

We begin by applying results of $\$ 4$ to point out that the answer for Question 2 is affirmative for phase spaces which are manifolds.

5.1. Theorem. Let $X$ be any metrizable manifold (with or without boundary) and let the components of $N(X)$ be compact. Then any connected subset of $E(X)$ is completely indivisible by 2.

Proof. This is an immediate consequence of Theorem 4.2(2).

Note that the requirement in (5.1) that $X$ be a manifold can be replaced by having $X$ be locally connected and locally compact. By Example 4.3 the compactness condition for components of $N(X)$ cannot be omitted.

5.2. Corollary. Let $X$ be any metrizable manifold. If $N(X)$ is totally dis. connected, then any connected subset of $N(X)$ is completely indivisible by 2.

Occasionally the connectedness of the set $E(X)$ is already provided by other conditions such as the one given in the following theorem.

5.3. Theorem. Let $X$ be a connected metrizable $n$-dimensional manifold $(n \geq 2)$ (with or without boundary). If $N(X)$ is 0-dimensional, then $2 \pitchfork E(X)$.

Proof. This follows immediately from (4.2(2)) and that no $(n-2)$-dimensional subset of any $n$-manifold disconnected the space. It is interesting to know that an alternate proof can be given by making use of (4.2(1)). By a theorem of Mazurkiewicz [9, Vol. 2, p. 466] the complement of any $(n-2)$-dimensional subset $A$ of Euclidean $n$-space is a semicontinuum. The statement can easily be general- 
ized to connected manifolds without boundary. For those with boundary, the complement of $A$ then has a dense subset which is a semicontinuum.

Clearly our argument shows that Theorem 5.3 holds also for any locally connected Cantor-manifold of dimension $\geq 2$.

To complete the discussion given after (1.5) we point out that it is also impossible to construct a transformation group on the plane so that $N(X)$ consists of rational pairs and its every orbit is bounded. This is due to the complete indivisibility of $E(X)$ and so $N(X)$ must be compact [10, Theorem 2].

5.4. Example. There exists a continuum $X$ in Euclidean 3-space and a continuous flow on $X$ such that $N(X)$ is countable, $E(X)$ is connected, but $2 \mid E(X)$.

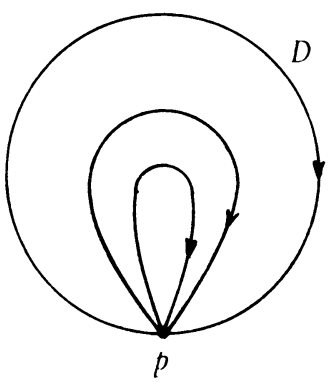

Figure (a)

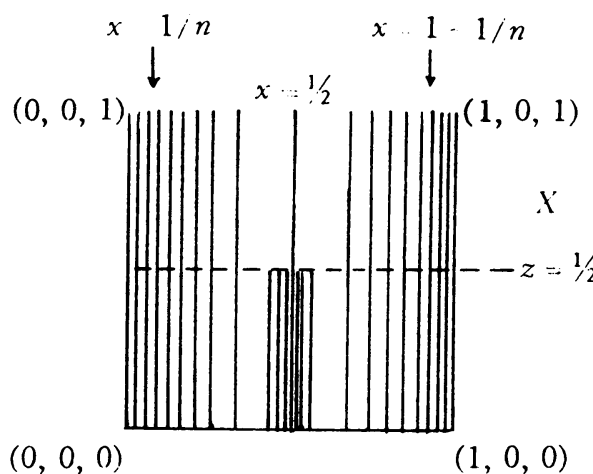

Figure (b)

We first construct a continuous flow on the unit 2-disk $D$ with a unique fixed point $p$ on the boundary (Figure (a)). The flow can be obtained rigorously by the following steps: (i) Construct a standard translation flow on the plane where the orbits are parallel to the $y$-axis. (ii) Project the flow of (i) on the Riemann sphere by the stereographic projection and add the fixed point $\{\infty\}$. (iii) Project the flow of (ii) on the $y \cdot z$ plane. The image of the projection is the disk $D$. (iv) Reflect the flow of (iii) with respect to the plane $z=1 / 2$. Note that $N(D)=\{p\}$.

We denote by $D_{n}$ the topological subdisk of $D$ which consists of all orbits whose $z$-coordinates are $\leq 1 / n(n=1,2,3, \cdots)$. The space $X$ will consist of the segment $I$ which joins the two points $(0,0,0)$ and $(1,0,0)$ and a countable family of sets $\left\{D_{n}\left(x_{i}\right)\right\}$, where $D_{n}\left(x_{i}\right)$ denotes the translate of $D_{n}$ along the $x$-axis from $(0,0,0)$ to the plane $x=x_{i}, 0 \leq x_{i} \leq 1$.

The family $\left\{D_{n}\left(x_{i}\right)\right\}$ is a countable union of sets $\left\{A_{n} \mid n=1,2,3, \ldots\right\}$ where the $A_{n}$ 's are defined by induction.

We first let $A_{1}=\bigcup\left\{D_{1}\left(x_{i}\right) \mid x_{i}=1 / n\right.$ or $\left.1-1 / n, n=2,3,4, \cdots\right\}$. Then $I \cup A_{1}$ is compact. For each point $x_{i}$ except 0 and 1 we construct a (distinct) sequence $\left\{x_{i j} \mid j=1,2,3, \cdots\right\}$ which converges from both sides to, but is disjoint from, $x_{i}$, and is sufficiently small in diameter, e.g. $\leq m_{i} / 4$, where $m_{i}$ is the minimum distance of $x_{i}$ from its two neighboring points. We let $A_{2}=\bigcup\left\{D_{2}\left(x_{i j}\right)\right\}$ $i, j\}$. Then $I \cup A_{1} \cup A_{2}$ is compact. For each point $x_{i j}$ we construct a sequence 
$\left\{x_{i j k} \mid k=1,2,3, \cdots\right\}$ converging from both sides to, but disjoint from, $x_{i j}$, and smaller in diameter, e.g. $\leq m_{i j} / 4^{2}$, where $m_{i j}$ is the minimum distance of $x_{i j}$ from its one or two neighboring points in the family $\left\{x_{i j}\right\}$ ( $i$ fixed). We let $A_{3}=$ $\bigcup\left\{D_{3}\left(x_{i j k}\right) \mid i, j, k\right\}$. Then $I \cup A_{1} \cup A_{2} \cup A_{3}$ is compact. We continue this process indefinitely to obtain the entire sequence $\left\{A_{n} \mid n=1,2, \cdots\right\}$. Then $X=I \cup$ $\left(\bigcup_{n=1}^{\infty} A_{n}\right)$ is a continuum. We let each point of $I$ be a fixed point and on each $D_{n}(x)$ the flow is defined as its horizontal translated copy $D_{n}$. This defines a continuous flow on $X$. Figure (b) is the projection of $X$ on the $x-z$ plane, where only $l$, the projection of $A_{1}$, and the projection of $\left\{D_{2}\left(x_{i j}\right) \mid j=1,2, \cdots ; x_{i}=1 / 2\right\}$ are shown.

It is easy to verify that $N(X)$ consists of exactly the intersections of $I$ with the attached disks. Hence $N(X)$ is countable. It is also not difficult to see that $E(X)$ is connected. Thus suppose $E(X)$ is decomposed into two disjoint relatively open nonempty sets $U$ and $V$. It is clear that if $I \cap E(X) \subset U$ then $V=\varnothing$. For if $V$ meets any $A_{n}$ then it meets all $A_{m}, n \leq m$, and a suitable sequence in $V$ can be chosen which converges to a point in the set $\operatorname{Cl}\left[\bigcup\left\{A_{n} \mid n=1,2,3, \ldots\right\}\right]$ $-\bigcup\left\{A_{n} \mid n=1,2,3, \ldots\right\} \subset I \cap E(X) \subset U$. Now by the construction, the point $5 / 12$, which is the midpoint of $1 / 3$ and $1 / 2$, is not in $\operatorname{Cl}\left[\bigcup\left\{A_{n} \mid n=1,2,3, \ldots\right\}\right]$. Hence we may assume that $U$ contains an open interval around $5 / 12$. Let $J=(a, 5 / 12]$ be the maximum half-open interval in $I$ such that $J \cap E(X) \subset U$. It is not difficult to see that $a=0$. We can draw a similar conclusion on the right-hand side of $5 / 12$. This gives $I \cap E(X) \subset U$, which is a contradiction. Hence $E(X)$ is connected.

In the previous example the set $N(X)$ is countable. This example can be used to construct a similar one such that $N(X)$ is uncountable and 0 -dimensional.

5.5. Example. There exists a continuum $X$ in Euclidean 4-space and a continuous flow on $X$ such that $N(X)$ is 0 -dimensional uncountable, $E(X)$ is connected, but $2 \mid E(X)$.

We denote the Cartesian coordinates of Euclidean 4-space, $\mathbf{R}^{4}$, by $(x, y, z, w)$. Let $X_{1}$ denote the continuum in Example 5.4. Consider the 2-dimensional $x-w$ plane $\Pi$ in $\mathbf{R}^{4}$. Then $\Pi \cap X_{1}=I$, where $I$ is the unit interval on the $x$-axis. We place the usual Cantor set on the segment which joins the two points $(1,1 / 2)$ and $(1,-1 / 2)$ in $\Pi$ so that the two points are also in the Cantor set. Call this resulting Cantor set $C$. For each $c=\left(x_{c}, w_{c}\right) \in C$ we let $P_{c}$ be the plane in $\mathbf{R}^{4}$ which is perpendicular to $\Pi$ and passes through the origin and the point $c$ in $\Pi$. For the point $c=(1,0) \in \Pi$ we simply denote $P=P_{c}$. Consider the linear transformation $f_{c}$ of $\mathbf{R}^{4}$ which leaves the $x, y$ and $z$ coordinates fixed and which takes $w$ to $w_{c} x$. The restriction of $f_{c}$ to $P$ is then a homeomorphism from $P$ onto $P_{c}$, hence it takes the continuous flow on $X_{1}$ to a continuous flow on $X_{1} f_{c}$. Now we simply let $X=\bigcup\left\{X_{1} f_{c} \mid c \in C\right\}$. The space $X$ is clearly compact. The continuous 
flow on $X$ is defined by its restrictions on the family $\left\{X_{1} f_{c} \mid c \in C\right\}$. It is not difficult to see that $N(X)=\bigcup\left\{N\left(X_{1} f_{c}\right) \mid c \in C\right\}$. Hence $N(X)$ is a subset of $\Pi$ which is exactly $\left(N\left(X_{1}\right) \times R\right) \cap \Lambda$, where $\Lambda$ is the set of all line segments joining 0 to $C$. The set $N(X)$ is 0 -dimensional and uncountable. We note that $\bigcap\left\{X_{1} f_{c} \mid c \in C\right\}$ $=D_{1}(0)$, where $D_{1}(0)$ is the disk in $X_{1}$ attached to the point 0 . Both $X$ and $E(X)$ are then connected, since each is a union of connected sets with at least one point in common.

The sets $N(X)$ in the two previous examples consist of fixed points. This is not surprising since the acting group is connected and so every component of the invariant set $N(X)$ must also be invariant. We now modify (5.5) to be a transformation group, whose acting group is $\mathbf{Z} \times \mathbf{Z}, \mathbf{Z}$ the set of integers, so that $N(X)$ has no fixed points.

5.6. Example. There exists a continuum $X^{\prime}$ in Euclidean 4-space and a transformation group $\left(X^{\prime}, \mathbf{Z} \times \mathbf{Z}, \pi\right)$ such that $N\left(X^{\prime}\right)$ is 0 -dimensional infinite, consisting of no fixed points, $E\left(X^{\prime}\right)$ is connected and $2 \mid E\left(X^{\prime}\right)$.

Again consider the set $X$ in Example 5.4. We let $X_{2}=\{(x, y, z) \in X \mid 5 / 12 \leq$ $x \leq 1\}$. It is easily verified that $X_{2}$ is an invariant subcotinuum of $X$ which has the same properties as stated in (5.4) for the set $X$. Consider the affine transformation $f$ given by $(x, y, z) f=((12 / 7) x-5 / 7, y, z)$. Then $f$ maps the interval $[5 / 12,7]$ of the $x$-axis onto the unit interval $[0,1]$. Let $X_{3}=X_{2} f$ and define a flow on $X_{3}$ by corresponding $f$ with points in $X_{2}$. Then $X_{3}$ has the same properties as the set $X$ stated in (5.4). Now in Example 5.5 we replace the set $X_{1}$ by the set $X_{3}$ and go through the same process to obtain a continuous flow on a continuum $X^{\prime}$, which has the properties as stated in (5.5). Note now $0 \notin N\left(X^{\prime}\right)$ and the intersection of $X^{\prime}$ with the $w$-axis consists of only the point 0 . Let $g$ be the homeomorphism of $X^{\prime}$ corresponding to the time 1 of the continuous flow.

Now there exists a discrete flow on the Cantor set $C$ so that $C$ becomes an equicontinuous, or equivalently uniformly almost periodic minimal set [3]. Let $b$ be the generator of this discrete flow. Let $\Pi$ again be the $x-w$ plane. Then $b$ is extendable to $X^{\prime} \cap \Pi$ by leaving points on every vertical line of $\Pi$ staying within the line. The next step is to extend $b$ to $X^{\prime}$ canonically. The final extension generates a discrete flow on $X^{\prime}$ which is uniformly equicontinuous. It is easy to see that the two homeomorphisms $g$ and $b$ commute. Take the abelian group $\mathbf{Z} \times \mathbf{Z}$ generated by $g$ and $b$. It is clear that the set $N\left(X^{\prime}\right)$ remains unchanged under the new acting group $\mathbf{Z} \times \mathbf{Z}$. Now the orbit closure of every point in $N\left(X^{\prime}\right)$ in the transformation group $\left(X^{\prime}, \mathbf{Z} \times \mathbf{Z}, \pi\right)$ is a minimal set which is homeomorphic to the Cantor set. Hence there are no fixed points in $N\left(X^{\prime}\right)$.

We now consider another case which concerns the possibility of a subset being $N(X)$ of a compact metric space $X$. We note that there is no continuous flow on 
the unit interval $X=[0,1]$ so that $N(X)$ is the set of rational points. For the set of fixed points $F$ of the flow is closed. If $F=X$, then $N(X)=\varnothing$. If $F \neq X$, then $E(X)$ contains an interval $(C X-F)$. There is also no discrete flow on $[0,1]$ so that $N(X)$ is the set of rational points. Thus let $(X, \mathbf{Z}, \pi)$ be a discrete flow, $\mathbf{Z}$ the set of integers. Then $(X, 2 Z, \pi)$ can be extended to a continuous flow. This is due to the fact that the generator of $(X, 2 Z, \pi)$ is a strictly increasing homeomorphism; hence it is embeddable in a continuous flow [2]. It is easy to verify that the set $N(X)$ for $(X, 2 Z, \pi)$ and that of its extended continuous flow are the same. Hence our previous argument on continuous flow applies. It is interesting to know that a slight extension of the interval can make the set of rational points be the set $N(X)$ in the interval $[0,1]$.

5.7. Example. There exists a continuous flow on a 1-dimensional Peano curve $X$ in the plane which is the extension of $[0,1]$ such that $N(X) \cap[0,1]$ consists of the set of rational numbers.

Define a function $f$ on $[0,1]$ by

$$
x f= \begin{cases}1 / q & \text { if } x \text { is rational } p / q \text { (in irreducible form and } q>0), \\ 0 & \text { if } x \text { is irrational, } \\ 1 & \text { if } x=0 .\end{cases}
$$

The function $f$ is well known to be continuous exactly at the irrational points. Let $X$ be the union of segments joining points of the graph of $f$ to their corresponding base points. It is easy to see that there is a continuous flow on $X$ so that $[0,1]$ in union with its graph forms the fixed-point set of $X$. Then $N(X)$ consists of exactly the set of rational points and its graph. The space $X$ is a compact, connected and locally connected set and is therefore a Peano curve.

If we change the function $f$ to $g$ by

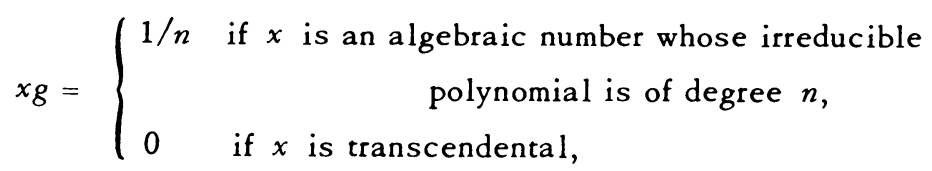

then the resulting space $X$ has the same topological properties as before, only now $N(X) \cap[0,1]$ consists of the set of algebraic numbers.

5.8. Remark. In considering the notion of indivisibility and sets which are admissible as an $N(X)$, it is important to hold the phase space $X$ to be locally compact and $T_{2}$. If either one of the two properties fails, many of our previous results and remarks can easily be disproved by simple counterexamples.

\section{REFERENCES}

1. N. Bourbaki, Éléments de mathématique. Part. 1. Les structures fondamentales de l'analyse. Livre III: Topologie générale, Actualités Sci. Indust., no. 1029, Hermann, Paris, 1947; English transl., Addison-Wesley, Reading, Mass., 1966. MR 9, 261; MR 34 \#5044b. 
2. M. K. Fort, Jr., The embedding of homeomorphisms in flows, Proc. Amer. Math. Soc. 6 (1955), 960-967. MR 18, 326.

3. W. H. Gottschalk, Minimal sets: an introduction to topological dynamics, Bull. Amer. Math. Soc. 64 (1958), 336-351. MR 20 \#6484.

4. W. H. Gottschalk and G. A. Hedlund, Topological dynamics, Amer. Math. Soc. Colloq. Publ., vol. 36, Amer. Math. Soc., Providence, R. I., 1955. MR 17, 650.

5. W. J. Gray and F.A. Roberson, On the near equicontinuity of transformation groups, Proc. Amer. Math. Soc. 23 (1969), 59-63. MR 39 \#7583.

6. T. Homma and S. Kinoshita, On homeomorphisms which are regular except for a finite number of points, Osaka Math. J. 7 (1955), 29-38. MR 16, 1140.

7. S. K. Kaul, On almost regular homeomorphisms, Canad. J. Math. 20 (1968), 1-6. MR $36 \# 5908$.

8. J. L. Kelley, General topology, Van Nostrand, New York, 1955. MR 16, 1136.

9. K. Kuratowski, Topologie, Vols. 1, 2, PWN, Warsaw, 1958, 1961; English transl., PWN, Warsaw; Academic Press, New York, 1966, 1968. MR 19, 873; MR 24 \#A2958; MR $36 \# 839$; MR $41 \# 4467$.

10. P.-F. Lam, On a theorem of B. von Kérekjárto, Bull. Amer. Math. Soc. 77 (1971), 230-234. MR $42 \# 2459$.

11. _- Almost equicontinuous transformation groups, Trans. Amer. Math. Soc. (to appear).

12. N. G. Markley, Transitive homeomorphisms of the circle, Math. Systems Theory 2 (1968), 247-249. MR 38 \#198.

13. W. Sierpinski, General topology, Math. Expositions, no. 7, Univ. of Toronto Press, Toronto, 1952. MR 14, 394.

14. L. A. Steen and J. A. Seebach, Jr., Counterexamples in topology, Holt, Rinehart and Winston, New York, 1970. MR $42 \# 1040$.

DEPARTMENT OF MATHEMATICS, UNIVERSITY OF MISSOURI, COLUMBIA, MISSOURI 65201

Current address: School of Mathematics, Institute for Advanced Study, Princeton, New Jersey 08540 